\title{
Repeat general anaesthesia for paediatric dentistry
}

\author{
M. Harrison, ${ }^{1}$ and L. Nutting, ${ }^{2}$
}

\begin{abstract}
Aims To investigate patterns of referral, disease and treatment for healthy children who had received two or more dental general anaesthetics (DGA) for exodontia.

Methods Records from 200 episodes of repeat DGA were studied retrospectively.

Results The mean age of patients at first referral was $5 \mathrm{y} 4 \mathrm{~m}$, and the mean interval before repeat was 22 months. Self-referrals rose from $14 \%$ at DGA1 to $30 \%$ at DGA2. New caries at second referral, where all diagnosed disease had been treated at DGA1, accounted for only $15 \%$ of the total. Where a specific request was made in the referral, only $8 \%$ of letters matched the charting made in hospital. $30 \%$ of all specific requests were honoured, but then required treatment for previously diagnosed caries at DGA2. 75\% of single-tooth extractions required repeat DGA for caries left at DGA1.

Conclusions It may be too optimistic to address only the most grossly diseased teeth when a child requires GA exodontia. A more radical treatment-planning approach, combining primary care, secondary care and public health considerations, may be required to avoid the unnecessary use of DGA.
\end{abstract}

$\mathrm{T}^{\mathrm{t}}$ is nearly ten years since the recommendation that 'the use of general anaesthesia should be avoided wherever possible' became an underlying principle of the provision of modern dental care in the UK. The subsequent upheavals in patterns of provision of care, particularly for the child patient, reverberate through all branches of the profession up to the present.

That 'general anaesthesia is a procedure which is never without risk $^{2}$ is axiomatic, but the risks are difficult to quantify and the technique may be extremely valuable; either for the very young or for those with extensive disease. ${ }^{3,4}$ The use of dental general anaesthesia (DGA) has been shown to be determined by factors other than clinical need, non-clinical factors including the availability or convenience of DGA facilities in a locality, the attitude of the dentist, and the overall public/parental attitudes to DGA. ${ }^{5}$ An apparent increase in the use of DGA in primary care, ${ }^{6} \mathrm{com}$ bined with a series of highly publicised child deaths, precipitated a change in GDC guideline ${ }^{2}$ which has now re-drawn the map as far as DGA is concerned in the UK. It is in this climate of the need for reduction of DGA that this study was undertaken, focusing on children who attend for a second DGA.

\footnotetext{
${ }^{1}$ Research Fellow in Paediatric Dentistry, Department of Orthodontics and Paediatric Dentistry, Guy's, King's and St Thomas' Dental Institute;

${ }^{2}$ Demonstrator in Paediatric Dentistry, Department of Orthodontics and

Paediatric Dentistry, Guy's, King's and St Thomas' Dental Institute

Correspondence to: $M$ Harrison, Department of Orthodontics and Paediatric Dentistry, Guy's, King's and St Thomas' Dental Institute, Floor 22 Guy's Tower, London Bridge, London SE1 9RT

email:mike.harrison@kcl.ac.uk

REFEREED PAPER

Received 15.07.99; accepted 17.01.00

() British Dental Journal 2000; 189: 35-37
}

\section{Materials and methods}

Demographic data concerning cases for DGA at Guy's Dental Hospital, London, were obtained retrospectively from a 5-year period (1992-1997). Children with a medical history liable to complicate a general anaesthetic were excluded, as were children receiving DGA for exodontia of sound teeth for orthodontic purposes. Repeat DGAs were identified, and the hospital records were sought. Many records were not retrievable, and after two searches of the stored records, it was decided to cease data collation after 200 had been processed. Radiographs were available in all cases. The data fields are described in Table 1.

Statistical analysis was carried out using SPSS. The Shapiro-Wilk test demonstrated normality, and Pearson's Rank test was used to identify correlation between variables.

\section{Table 1 Data collected}

$\begin{array}{ll}\text { - Date of 1st referral } & \text { - Date of 2nd referral } \\ \text { - Source of 1st referral } & \text { - Source of 2nd referral } \\ \text { - Referrer's request 1 } & \text { - Referrer's request 2 } \\ \text { - Charted disease 1 } & \text { - Charted disease 2 } \\ \text { - Teeth extracted at DGA1 } & \text { - Teeth extracted at DGA2 }\end{array}$

\section{Results}

Over the 5-year period, 3,872 children were seen for simple exodontia under general anaesthesia. From these, 369 repeats were identified. Data collection was stopped when the first available 200 hospital files had been retrieved.

\section{Source}

While for both episodes, the main referral source was the GDS, Table 2 illustrates the change in source between DGA1 and DGA2.

Table 2 Source of referral

\begin{tabular}{lcccc}
\hline & DGA1 & $\%$ & DGA2 & $\%$ \\
\hline GDS & 142 & $71 \%$ & 112 & $56 \%$ \\
CDS & 24 & $12 \%$ & 28 & $14 \%$ \\
Self-referred & 34 & $17 \%$ & 60 & $30 \%$ \\
\hline
\end{tabular}

Age

The mean age of patients at first referral was $5 y 4 \mathrm{~m}$, the range being $1 \mathrm{y} 5 \mathrm{~m}$ to $12 \mathrm{y} 3 \mathrm{~m}$.

Interval

The interval between GA1 and GA2 ranged from 42 days to $4 y 7 \mathrm{~m}$, with a mean of $1 \mathrm{y} 9 \mathrm{~m}$.

Quality of referrals

A specific request was made in $75 \%$ of written referrals, the remainder being to 'see and treat'. In only $8 \%$ of referral letters did the 
referrer's request mention all disease diagnosed at the pre-assessment clinic. Despite this, in $31 \%$ of all cases where a specific request was made, it was honoured; the remaining disease being left untreated.

\section{Single-tooth extractions}

Single-tooth extractions were requested in 53 cases, $75 \%$ of these then requiring a subsequent DGA for other disease diagnosed but untreated at DGA1.

\section{Residual disease}

Although the proportion of carious teeth extracted was significantly greater at DGA1 than DGA2 $(P<0.0001)$, on average $31 \%$ of carious teeth diagnosed at DGA2 were then left. Residual disease levels are illustrated in Table 3. The records included 28 children who had received 3 DGAs and 5 children who had received 4 DGAs.

Positive correlation was found between the number of teeth extracted at GA1 and the time interval before the repeat DGA (Pearson's correlation coefficient $=0.18, P<0.01$ ). Negative correlation was found between the number of carious teeth left at GA1 and the time interval before the repeat DGA (Pearson's correlation coefficient $=-0.20, P<0.01$ ).

Table 3 Summary of disease and treatment at DGA1 and DGA2

\begin{tabular}{lccc}
\hline & Mean & Mode & Range \\
\hline Carious teeth at DGA1 & 7.2 & 7 & $1-19$ \\
Teeth extracted at DGA1 & 4.2 & 4 & $1-14$ \\
Interval DGA1-DGA2 & $1 \mathrm{y} 9 \mathrm{~m}$ & - & $1.5 \mathrm{~m}-4 \mathrm{y} 7 \mathrm{~m}$ \\
Carious teeth at DGA2 & 5.1 & 5 & $1-16$ \\
Teeth extracted at DGA2 & 3.5 & 3 & $1-14$ \\
\hline
\end{tabular}

\section{Discussion}

This study raises three questions, which need to be addressed separately:

1. Should the referral centre communicate with the referrer in every case to clarify the requested treatment, and to discuss what measures should be taken to address any additional dental disease?

2. For the pre-cooperative child, the un-cooperative child, or the child who has had no previous experience of restorative dentistry, what should be done with restorable teeth if DGA is indicated for relief of acute symptoms?

3. If the circumstances of DGA are not an appropriate setting for preventive advice, how can children who have already had one DGA be efficiently and effectively targeted for preventive advice which may help avoid a repeat?

GDC guidelines state that it is the responsibility of a dentist when accepting a referral letter to ensure that the request is fully understood'. ' If the request is not felt to be appropriate, 'there is an obligation on the dentist to discuss the matter, prior to commencing treatment, with the referring practitioner and the patient'. This may provide the key to answering the question of what to do with the diseased teeth not mentioned in a referral letter. Communication with the referring dentist removes any ambiguity regarding the child's ability and the dentist's willingness to restore any potentially saveable teeth. The dentist accepting the referral for DGA usually has no prior knowledge of the child's ability to cope with restorative care. To send a child back to the referrer for restorations after extraction of a few unsaveable teeth is, in our opinion, poor judgement based on minimal information about the child, their parents, and the dentist with whom they are registered. This judgement, perhaps based on optimism and a wish to save teeth, accounted for $85 \%$ of repeat DGAs in our study.
The clinical dilemma faced by providers of DGA is typified by the pre- or un-cooperative child where the referral request is specifically for extraction of teeth causing pain/sepsis, but where other carious teeth are restorable. Pre-anaesthetic clinics, where the specific referral request is viewed in the light of the diagnosed disease, have been found to reduce the need for repeat DGA within 1 year. $^{8}$ Poor treatment planning has been identified as a contributary factor to repeat DGA. ${ }^{9}$ More aggressive prescribing of extractions, particularly in children under four, has been advocated by some to reduce repeats. ${ }^{10}$ Indeed this view is supported by this study, where positive correlation was found between the number of teeth extracted and the length of the interval before DGA2, and where $75 \%$ of single-tooth extractions required repeat DGA for caries diagnosed at DGA1.

Full-mouth rehabilitation under intubated GA may be a more positive solution to the problem. To the child it is no more emotionally traumatic than a short GA for exodontia. However, there may be increased risks from the anaesthetic procedure. Musclerelaxant drugs are required for intubation, duration of anaesthesia is prolonged, as may be the recovery time. Against these risks is balanced the knowledge that after comprehensive rehabilitation the need for further dental treatment is minimised in the short-term, allowing preventive strategies to be put in place.

When all diagnosed disease was dealt with at DGA1, our study demonstrated $15 \%$ of repeat DGAs had developed new disease. This signals a missed opportunity for disease prevention following DGA. This may be seen as a wider public health issue, where use of professionals complementary to dentistry and indeed professionals outside dentistry, such as health visitors, should be considered. ${ }^{11,12}$

While it is widely accepted that GA is sometimes necessary for the delivery of paediatric dental care, ${ }^{13}$ the authors take the view that repeat DGA is unacceptable and preventable. Exposing a child to a repeat DGA doubles the exposure to potential serious risk, no matter how small the probability. But perhaps an equally important reason for wishing to avoid repeat DGA is that the experience rarely does anything to enable a child to 'cope' with future dental care. Indeed the negative impression left by the experience may leave a child in a position where they may be even less amenable to dental care. ${ }^{14}$

That many parents consider DGA to be an acceptable method of delivery of paediatric dental care was reflected in our study, which showed that the number of self-referrals rose from $17 \%$ at DGA 1 to $30 \%$ at DGA2. When discussing the use of DGA with a parent, it is a dentist's responsibility to discuss both the useful and the undesirable aspects of DGA. The difficulty lies in balancing the two sides of the issue in an informed and consistent manner, while helping parents to find the most appropriate form of dental treatment for their own child.

Seeking to minimise the risk of repeat DGA is an important consideration in treatment planning for the child referred for their first DGA. While this study may have identified problems particular to the hospital in question with regard to the incidence of repeat DGA, discussion with other providers of paediatric DGA suggests that this is not the case. Indeed the Annual Report of Glasgow Dental Hospital 1997-1998 stated 'around 5000 children come to our Hospital each year for multiple extractions under general anaesthesia. At least $25 \%$ will return within the same year for further extractions'.

\section{Summary}

This was a study of identified repeat DGAs; a study which suggests the need for changes in the provision of DGA for exodontia in this centre. The motive for publishing the study is to encourage consideration toward reducing the incidence of repeat DGA in all centres where it is provided. In the meantime, children who require DGA should be regarded as a group who are vulnerable to repeat, and as 
such have a high dental need. When discussing inequalities in children's caries experience, Pitts recently recommended that integrated approaches to prevention should be focused on those children with the greatest need. ${ }^{15}$ Finding effective strategies for achieving concordance between parental and professional attitudes toward prevention of dental disease in children remains the key to addressing the circumstances leading to DGA, and repeat DGA in particular.

The authors would like to thank Mrs J Edmead for her invaluable assistance in retrieving the records used in this study.

1 Poswillo D E. General anaesthesia, sedation and resuscitation in dentistry: report of an expert working party. London: Department of Health, 1990.

2 General Anaesthesia and Resuscitation 4.8. General Dental Council: Maintaining Standards. November 1997 (revised May 1999).

3 Holt R D. Editorial. Int J Paediatr Dent 1999; 9: 1.

4 Holt R D, Rule D C, Davenport E S, Fung D E. The use of general anaesthesia for tooth extraction in children in London: a multi-centre study. Br Dent J 1992; 173: 333-339.

5 Hastings G B, Lawther S, Eadie D R, Haywood A, Lowry R, Evans D. General anaesthesia: who decides, and why? Br Dent J 1994; 177: 332-334.

6 Watt R G, Fuller S S. Oral health promotion-opportunity knocks! Br Dent J 1999; 186: 3-6.
7 What the patient expects 3.4. General Dental Council: Maintaining Standards. November 1997 (revised May 1999).

8 Grant S M, Davidson L E, Livesey S. Trends in exodontia under general anaesthesia at a dental teaching hospital. Br Dent J 1998; 185: 347-352.

9 Podesta J R, Watt R G. A quality assurance review of the patient referral process and user satisfaction of outpatient general anaesthesia services for dental treatment. Community Dent Health 1996; 13: 228-231.

10 Landes D P, Bradnock G. Demand for dental extractions performed under general anaesthesia for children by Leicestershire Community Dental Service. Community Dent Health 1996; 13: 105-110.

11 Hunter M L, Hood C A, Hunter B, Kingdon A. Oral health advice: reported experience of mothers of children aged 5 years and under referred for extraction of teeth under general anaesthesia. Int J Paediatr Dent 1998; 8: 13-17.

12 Quinn G, Freeman R. Dental health. 2. Working together in dental health education. Health Visit 1994; 67: 90-91.

13 Murray J J. General anesthesia and children's dental health: present trends and future needs. Anesth Pain Control Dent 1993; 2: 209-216.

14 Bridgman C M, Ashby D, Holloway P J. An investigation of the effects on children of tooth extraction under general anaesthesia in general dental practice. Br Dent J 1999; 186: 245-247.

15 Pitts N B. Inequalities in children's caries experience: the nature and size of the UK problem. Community Dent Health 1998; 15 Suppl 1: 296-300. 